\title{
SPARQLGPViz: SPARQL Graph Pattern Visualization $^{\star}$
}

\author{
Peb R. Aryan ${ }^{10000-0002-1698-1064]}$ and Fajar J. Ekaputra ${ }^{10000-0003-4569-2496]}$ \\ TU Wien, Favoritenstrasse 9-11 A-1040 Vienna, Austria \\ \{peb.aryan, fajar.ekaputra\}@tuwien.ac.at \\ http://semsys.ifs.tuwien.ac.at/
}

\begin{abstract}
We present SPARQLGPViz, a tool to visualize graph patterns in a SPARQL Query. The initial implementation of this tool displays exemplary SPARQL query using visual representation to identify the variables and their relationship for didactic purposes (i.e. teaching how to query using SPARQL).
\end{abstract}

Keywords: SPARQL · Graph Pattern · Visualization.

\section{Introduction}

Recently, there are growing numbers of RDF data available via SPARQL endpoints. The target users for these publicly available endpoints are typically nontechnical users. In doing so, data publishers usually provide not just the SPARQL endpoint service itself but also learning materials for new users who may not be familiar with SPARQL and RDF. To this end, a common approach for teaching SPARQL to these users is by introducing the its features in gradually increasing complexity along with examples of how some queries are formulated from the information need described in natural language ${ }^{1}$.

Despite the availability of SPARQL learning material, learning all aspects of the query language takes considerable time. Some people prefer to browse through the example queries and try to understand what data are requested using those queries. In this case, there is a need for an instrument to help understanding a SPARQL query without any prior information, e.g., using a graphical representation of the SPARQL query. In the literature, tools to help explain a SPARQL query are usually embedded within query builder tool $[1,2, ?, 4]$ or bound to a particular endpoint [3].

We aim to address this gap for SPARQL query visualization with SPARQLGPViz. SPARQLGPViz is developed to solve this problem without making any assumptions about the endpoint in which the query will be executed. This tool accepts as input a text file containing a valid SPARQL 1.1 query and generates

\footnotetext{
* This work was sponsored by the Austrian Research Promotion Agency FFG under grant 877389 (OBARIS). The authors thank the funder for its generous support.

${ }^{1}$ https://www.wikidata.org/wiki/Wikidata:SPARQL_tutorial
} 
an image file which depicts the terms involved in the query in a graphical manner thus making the query easier to grasp than in its written format.

The following sections describe how the SPARQL graph pattern is mapped to a visual representation (Section 2) and provide an example of how an example query is visualized (Section 3). Finally, we provide closing remarks identifying the open issues and planned future developments.

\section{Graph Pattern Visualization}

Graph pattern matching is the basis of SPARQL queries. A graph pattern contains RDF Triples with additional terms such as variables and/or property paths to be matched with the queries data.

\subsection{SPARQL Graph Pattern}

The basic syntax description of graph pattern consists of either an empty group or a set of triple patterns. The W3C recommendation of SPARQL 1.1 Query language[5] specifies how complex queries can be decomposed into combination of smaller patterns such as : 1. Basic Graph Patterns, where a set of triple patterns must match 2. Group Graph Pattern, where a set of graph patterns must all match 3. Optional Graph patterns, where additional patterns may extend the solution 4. Alternative Graph Pattern, where two or more possible patterns are tried 5. Patterns on Named Graphs, where patterns are matched against named graphs. The different patterns are assigned different colors to show how the triples are connected together. For example, triples that are optional and triples coming from another endpoints (i.e. through federation) can be easily distinguished.

\section{$2.2 \quad$ Visual Representation}

The graph pattern is represented using a directed graph (digraph). The subject and object part of the RDF Triple is represented as node and the predicate or property part is represented as edge with arrow head. The appearance of the nodes differ based on the type of the terms, as follows:

- URI nodes are displayed as ellipses with solid outline.

- Variable type nodes are displayed as ellipses with dashed outline.

- Blank Nodes are displayed as ellipses with dotted outline.

- Literals are displayed as box with solid outline.

In case of explicit value binding to variables (i.e. using VALUES clause), the values are displayed as boxes and connected to the bound variable through a dashed line. Different groups of triples are assigned to different colors to help differentiating different type of graph pattern such as optional, unions or subqueries and federated queries. 


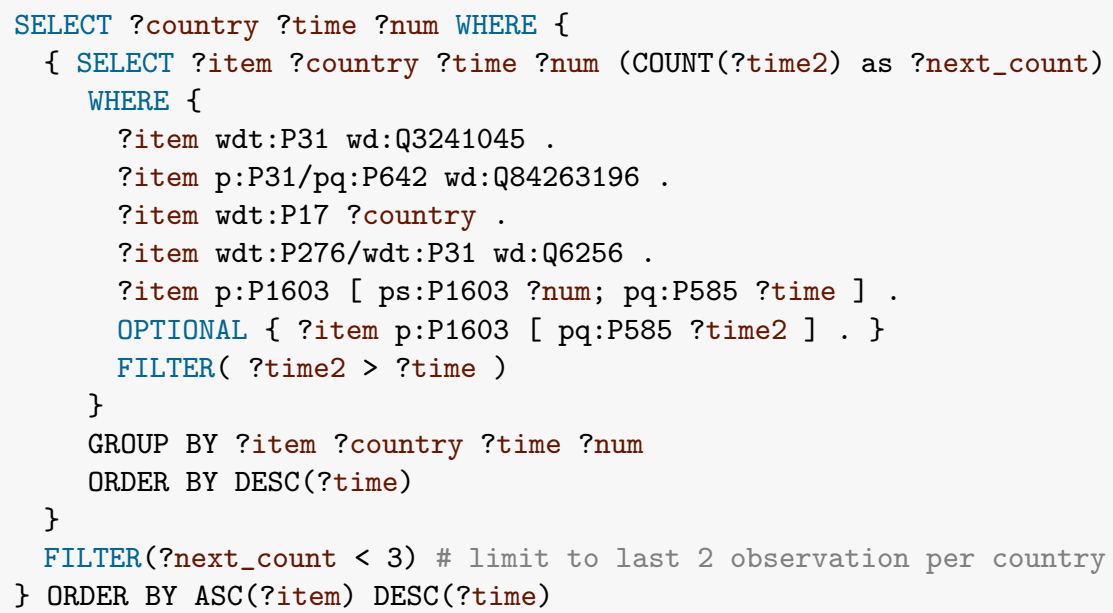

Fig. 1. Example SPARQL query on Wikidata containing property path and blank nodes.

The label of each node is the most compact textual representation whenever possible. In case of URIs, the label is a prefixed name if the prefixes are available in the prologue part of the SPARQL or via a text file supplied into additional parameter in the command-line. A property path is treated as a single label which consists of a combination of the path elements (i.e. URI or wildcard symbols).

\section{Demo}

As an example, the query shown in Figure 1 contains all mentioned term types (URI, Variable, and Blank Node). Additionally, it goes beyond containing just a basic graph pattern to contain a group graph pattern with a sub-query (line 2) and an optional graph pattern (line 9). The visualization of this query is shown in Figure 2.

The tool is implemented as command-line python scripts which can be executed via a terminal. The only dependencies are rdflib ${ }^{2}$ for parsing SPARQL 1.1 query and pygraphviz ${ }^{3}$ for wrapping graphviz tool suite which provides graph visualization representation (DOT language) and image generation tool. SPARQLGPViz code is publicly available ${ }^{4}$ using MIT License.

\footnotetext{
${ }^{2}$ https://pypi.org/project/rdflib/

3 https://pygraphviz.github.io/

${ }^{4}$ https://github.com/pebbie/sparqlgpviz
} 


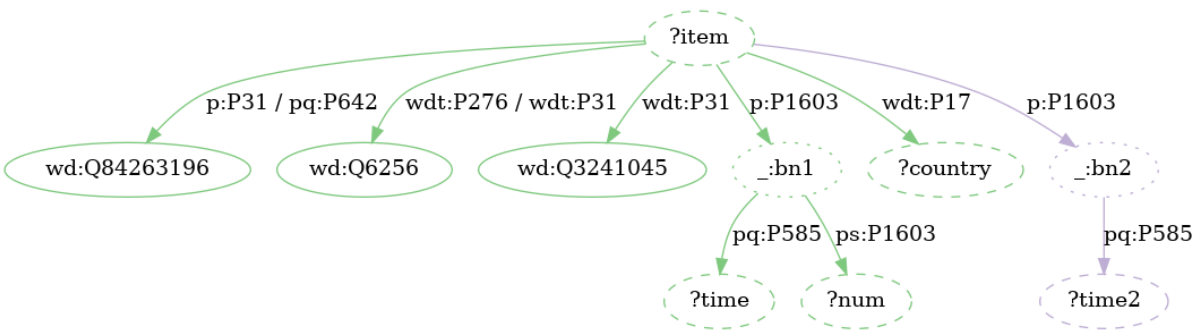

Fig. 2. Visualization result of the example SPARQL query

We use queries taken from a presentation slide ${ }^{5}$ that were used in a SPARQL Workshop ${ }^{6}$. The slide contains 12 SPARQL queries which access biological data from the resources of Swiss Institute of Bioinformatics. The queries and their visualization are included in the Github repository of SPARQLGPViz ${ }^{7}$.

\section{Future works}

The current implementation only covers the variables that are explicitly involved in the graph pattern while new variables derived from aggregation functions or binding declarations are not yet included in the output. These will be covered as part of future work. In addition, the tool can also show mapping or change of vocabulary in case of construct queries.

\section{References}

1. Cuddihy, Paul, Justin McHugh, Jenny Weisenberg Williams, Varish Mulwad, and Kareem S. Aggour.: SemTK: A Semantics Toolkit for User-friendly SPARQL Generation and Semantic Data Management. In International Semantic Web Conference (P\&D/Industry/BlueSky). 2018.

2. Čerāns, Kārlis, Agris Šostaks, Uldis Bojārs, Juris Bārzdiņš, Jūlija Ovčiņņikova, Lelde Lāce, Mikus Grasmanis, and Artūrs Sproǵis.: ViziQuer: a visual notation for RDF data analysis queries. In Research Conference on Metadata and Semantics Research, pp. 50-62. Springer, Cham, 2018.

3. Haag, Florian, Steffen Lohmann, Stephan Siek, and Thomas Ertl. : QueryVOWL: Visual composition of SPARQL queries. In European Semantic Web Conference, pp. 62-66. Springer, Cham, 2015.

4. Vega-Gorgojo, Guillermo, Martin Giese, Simen Heggestøyl, Ahmet Soylu, and Arild Waaler. "PepeSearch: semantic data for the masses." PloS one 11, no. 3 (2016): $\mathrm{e} 0151573$.

5. W3C : SPARQL 1.1 Query Language, https://www.w3.org/TR/sparql11-query/. Last accessed 13 Aug 2020

\footnotetext{
${ }^{5}$ https://edu.isb-sib.ch/mod/resource/view.php?id=4260

${ }^{6}$ https://edu.isb-sib.ch/course/view.php?id=440

${ }^{7}$ https://github.com/pebbie/sparqlgpviz/blob/master/rhea/rhea.md
} 